\title{
Comparison of a Portable and Non-Portable Ultrasound Machine in the Evaluation of Children with Sickle Cell Disease: A Pilot Study
}

\author{
Mohammed J. Alsaadi, MSc, $\mathrm{PhD}^{1 *}$; Joanne Widdup, BSc,MSc'; \\ Mohammed F. Aslam, MSc, $\mathrm{PhD}^{3}$; Subarna Chakravorty, MBBS, $\mathrm{PhD}^{4}$ \\ ${ }^{1}$ Radiology and Medical Imaging Department, College of Applied Medical Sciences, \\ Prince Sattam Bin Abdulaziz University, Alkharj, Saudi Arabia \\ ${ }^{2}$ Vascular Department, University Hospitals of Leicester NHS Trust, \\ Leicester, United Kingdom \\ ${ }^{3}$ Department of Surgery \& Cancer, Imperial College, London, United Kingdom \\ ${ }^{4}$ Hematology Department, King's College Hospital NHS Trust, London, United Kingdom
}

\begin{abstract}
Background: Transcranial color Doppler (TCCD) ultrasound is used to identify children with sickle cell disease (SCD) at high risk of developing stroke. There is anecdotal evidence to suggest that different ultrasound equipment can give different blood flow velocities. The purpose of this study was to compare two different TCCD ultrasound machines.

Methods and Results: A flow phantom was used to compare PSV measurements from a Philips IU-22 and Zonare Z-One ultrasound machine. Twenty-five children with SCD (aged between 2 and 16 years) attending the outpatient clinic at St. Mary's Hospital, Imperial College Healthcare NHS Trust, as part of the NHS Sickle Cell \& Thalassaemia (SC\&T) screening program were studied. The two ultrasound machines compared the TAMM velocities in the middle cerebral artery and stroke risk categorization. PSV measurements using a flow phantom were underestimated by Philips IU-22 (31\%) and Zonare Z-One (53\%). TAMM velocities varied considerably between machines, with a poor agreement in stroke risk categorization. As a result, three children identified at increased risk of stroke by Philips IU-22 were not identified by Zonare Z-One.

Conclusion: Two ultrasound machines were found to underestimate PSV using a flow phantom. The two ultrasound machines were shown to positively correlate, and this was statistically significant. However, there was variation in the TAMM velocities recorded by the machines which resulted in the different categorization of the stroke risk of a small number of the subjects. This pilot study confirms the feasibility and clinical significance of this investigation.(International Journal of Biomedicine. 2021;11(4):435-440.)
\end{abstract}

Key Words: sickle cell disease $\bullet$ stroke $\bullet$ ultrasound $\bullet$ transcranial Doppler

For citation: Alsaadi MJ, Widdup J, Aslam MF, Chakravorty S. Comparison of a Portable and Non-Portable Ultrasound Machine in the Evaluation of Children with Sickle Cell Disease: A Pilot Study. International Journal of Biomedicine. 2021;11(4):435-440. doi:10.21103/Article11(4)_OA7

\section{Abbreviations}

MCA, middle cerebral artery; SCD, sickle cell disease; PSV, peak systolic velocity; TCD, transcranial Doppler; TCCD, transcranial color Doppler; TAMM, time-averaged maximum mean.

\section{Introduction}

Sickle cell disease (SCD) is a genetic disorder arising from a point mutation in the gene encoding the hemoglobin protein beta-globin. When inherited in homozygosity or compound heterozygosity with other relevant beta-globin mutations, the resultant mutant hemoglobin causes the development of the clinical syndrome. These genetic abnormalities result in anemia, intermittent severe pain, susceptibility to infection, chronic damage to lungs, joints, kidneys and other bodily 
organs, and reduction in life expectancy. ${ }^{(1)}$ Additionally, up to $11 \%$ of patients with SCD develop strokes by age 20 , with the highest risk of ischemic strokes in HbSS genotype. ${ }^{(2)}$

Screening for stroke risk can be undertaken using transcranial Doppler vascular ultrasound, either by non-imaging (TCD) or transcranial color Doppler (TCCD) ultrasound. ${ }^{(3)}$ These are non-invasive scans that allow real-time evaluation of the intracranial cerebral circulation by insonation of the cerebral circulation via transcranial windows that include studying the blood flow velocities and the presence of turbulence or stenosis within arteries. A TCD ultrasonography in SCD utilizes the temporal window to examine the middle, anterior and posterior cerebral arteries (MCA, ACA, and PCA, respectively) and distal internal carotid arteries. TAMM velocities greater than $200 \mathrm{~cm} / \mathrm{s}$ in all arteries are considered abnormal (except in ACA, where a lower threshold of $>170 \mathrm{~cm} / \mathrm{sec}$ is considered abnormal). Readings below $170 \mathrm{~cm} / \mathrm{sec}$ are normal and those between $170-199 \mathrm{~cm} / \mathrm{sec}$ are termed conditional. ${ }^{(4)}$

A large, randomized, controlled study, The Stroke Prevention Trial in Sickle Cell Anaemia, demonstrated the clinical utility of chronic transfusion therapy in children with abnormal TCD readings in primary stroke prevention. ${ }^{(4)}$ This led to the uptake of routine TCD screening in many clinical SCD programs. Several reports indicate that stroke prevention programs using TCD surveillance and transfusion therapy in at-risk children have reduced the incidence of childhood stroke in SCD from $11 \%{ }^{(2)}$ to $1.9 \%$ by 18 years. ${ }^{(5)}$ Clinical and cost-effective data in the UK context provide further support for screening programs for stroke risk assessment. ${ }^{(6)}$

The UK Forum on Haemoglobin Disorders (UKFHD) has recommended that UK SCD centers offer annual TCD scans to children aged 2-16 with severe $\mathrm{SCD}\left(^{(7)}\right.$ Another critical document published by UKFHD, the Quality StandardsHealth Services for People with Haemoglobin Disorders, also stipulate the need for adequate training, maintenance of competencies, and quality assurance of the TCD screening program. ${ }^{(8)}$ Both imaging and non-imaging Doppler techniques are used in this context, based mainly on the local availability of suitable scanners. Several studies have compared TCD with $\mathrm{TCCD},{ }^{(9)}$ and the current consensus is to use the same arterial velocity cut-offs in either scanning modality. ${ }^{(7)}$ To ensure universal uptake of TCD screening, many Primary Treatment Centers for SCD in the UK have developed outreach TCD surveillance programs in collaboration with local clinical teams to provide TCD screening closer to the patients' homes. (10) These are usually undertaken by using portable vascular ultrasound machines.

Whilst several studies have compared imaging versus non-imaging vascular ultrasound scans in SCD, no studies exist that indirectly compare a portable TCD machine with a non-portable one. Both are used within our SCD program, and it was essential to ensure that comparable readings were achieved using these machines. This study aimed to directly compare TCD readings using the available scanners within our service - the non-portable Philips IU-22 scanner with an S5-1 phased array transducer $(5.0-1.0 \mathrm{MHz})$ and portable Zonare Z-One scanner with a P4-1C phased array transducer (4.0$1.8 \mathrm{MHz}$ ). We hypothesized that velocity measurements from the portable ultrasound machine would not be significantly different from those obtained by the non-portable laboratorybased machine.

\section{Methods}

The study design was a prospective study. Patients with SCD, aged between 2 and 16 years, attending our tertiary pediatric hematology clinic for stroke surveillance, were invited and eligible for the study. Based on patient numbers within the service, we aimed to study 25 consecutive patients referred to the vascular sciences department for routine TCD scanning. This study was approved by the National Research Ethical service, UK Ref number (Ref 13/LO/1503). This study was also registered at the CT.gov, ref number (NCT02090881); written informed consent was obtained from legal guardians. National Research Ethical service permission was obtained for the study.

Study participants were scanned by a single operator who has more than 5 years of experience with imaging TCCD. Initially, a full TCD assessment was performed using the nonportable Philips IU-22 with an S5-1 phased array transducer $(5.0-1.0 \mathrm{MHz})$. The measurement was repeated in every patient in only the MCA on each side of the head using the portable Zonare Z-One scanner with a P4-1C phased array transducer $(4.0-1.8 \mathrm{MHz})$. For both examinations, a US standardized screening protocol was used, recording velocities from the transtemporal windows, a $5 \mathrm{~mm}$ sample volume size, with no angle correction. The gain was adjusted to see the spectral Doppler velocity waveform without noise in the background. For this study, the TAMM velocities were compared because stroke risk categorization, according to the STOP trial, was based on TAMM velocity, not peak systolic or end-diastolic velocities. Each study was categorized as normal if the TAMM velocity $<155 \mathrm{~cm} / \mathrm{sec}$, conditional if the TAMM velocity was $155-179 \mathrm{~cm} / \mathrm{sec}$ and abnormal or high risk if TAMM velocity $\geq 180 \mathrm{~cm} / \mathrm{sec}$, or inadequate according to criteria previously developed by the STOP protocol. The participants were not permitted to sleep during the scan to avoid an increase in blood carbon dioxide levels in sleep, which can increase in TCD velocities.

A pulsatile flow-simulating phantom (Fig.1), which generates pulsatile flow from a computer-controlled pump, was used to eliminate patient variability. The manufacturer had calibrated the speed to $120 \mathrm{~cm} / \mathrm{sec}$. The flow simulator was used to pump blood-mimicking fluid through a vessel surrounded by an agar-based, tissue-mimicking material. A glycerol/water mix was used to give a liquid with a density and viscosity close to human blood to mimic the speed of sound of $1540 \mathrm{~m} / \mathrm{sec}^{\text {;(11) }} 850 \mathrm{ml}$ of water was mixed with 150 $\mathrm{ml}$ glycerol. PSV was measured using the Philips IU-22 nonportable scanner with an S5-1 phased array transducer (5.0$1.0 \mathrm{MHz}$ ) and a Zonare Z-One portable scanner with a P4-1C phased array transducer $(4.0-1.8 \mathrm{MHz})$. The measurement of PSV was repeated 25 times with each transducer and the mean PSV was calculated. No angle correction was used with the phased array transducers with a sample volume of $5 \mathrm{~mm}$, as per the SCD screening protocol. 


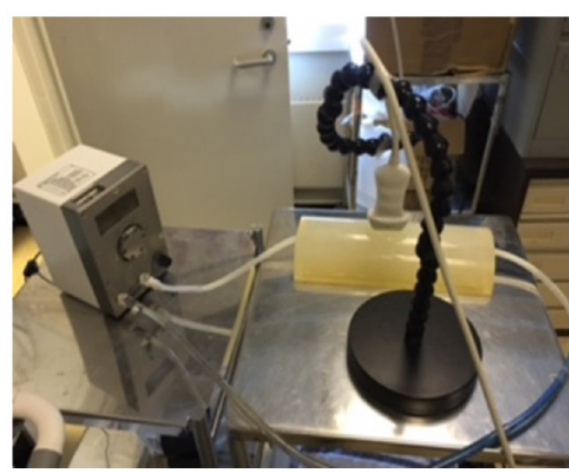

Fig 1. Pulsatile flow-simulating phantom design for the measurement's accuracy.

Statistical analyses were performed using SPSS Statistics version 21 (IBM). Spearman's correlation coefficients were calculated to assess the relationships between different parameters in order to assess the strength of the association between the two measures. Intraclass coefficient correlation (ICC) and 95\% confidence intervals (CI) were used to measure the strength of the association between the measurements from both pieces of equipment and therefore the validity of the measurement. It has been stated that ICC $>0.75$ implies acceptable validity. ${ }^{(12)}$ Bland-Altman plots were used to estimates the agreement between TAMM velocity measurements by determining how much the velocity from one machine differs from another. The level of statistical significance was defined as $P<0.05$.

\section{Results}

Twenty-five children were included in the study; all had HbSS genotype. The children underwent routine steadystate laboratory tests on the day of their scan or within two weeks. On the day of the scan, all children were reviewed by a clinician as part of their routine clinical assessment. This ensured that they were well at the time of the scan. Table 1 presents demographic details of study participants.

Table 1.

Demographic details of study participants

\begin{tabular}{lcc}
\hline Genotype & HbSS & N \\
\hline Female & 25 \\
\hline Median age in years (IQR) & $9(6-13)$ & 14 \\
\hline Median Haemoglobin in g/l (IQR) & $83(76.5-90.5)$ & 25 \\
\hline Median Height in cm (IQR) & $134.5(116.8-149.1)$ & 22 \\
\hline Median weight in kg (IQR) & $27.4(19.8-38.8)$ & 23 \\
\hline
\end{tabular}

IQR: Interquartile range

Table 2 outlines the TAMM velocities recorded in the right and left MCA using both scanners. The data demonstrated a significant negative correlation between age and TAMM velocities in the left MCA using the Philips IU-22. There was no statistically significant correlation between the hemoglobin concentration and recorded TAMM velocity. Adequate tracings were obtained from 23 of 25 examinations of the right MCA and 25 of 25 examinations of the left MCA. This was because two of the subjects had poor right temporal windows, and so velocities were not recorded from the MCA. It was noted that the MCA was more difficult to visualize and obtain an optimal signal with the Zonare Z-One because of the limitations of the size of the screen and the resolution.

\section{Table 2.}

TAMM velocities recorded in the right and left MCA using Philips IU-22 and Zonare Z-One

\begin{tabular}{|c|c|c|c|c|c|}
\hline & \multirow[b]{2}{*}{$N \quad \begin{array}{c}\text { Median } \\
\text { (IQR) }\end{array}$} & \multicolumn{2}{|c|}{$\begin{array}{l}\text { Spearman's } \\
\text { correlation } \\
\text { with age }\end{array}$} & \multicolumn{2}{|c|}{$\begin{array}{c}\text { Spearman's } \\
\text { correlation } \\
\text { with Hb levels }\end{array}$} \\
\hline & & rho & $P$ & rho & $p$ \\
\hline $\begin{array}{l}\text { Philips Right MCA } \\
\text { (TAMM) }\end{array}$ & $23 \begin{array}{c}119 \\
(110-128)\end{array}$ & -0.425 & 0.043 & -0.111 & 0.651 \\
\hline $\begin{array}{l}\text { Zonare Right MCA } \\
\text { (TAMM) }\end{array}$ & $23 \begin{array}{c}105 \\
(95-116)\end{array}$ & -0.462 & 0.027 & 0.258 & 0.235 \\
\hline $\begin{array}{l}\text { Philips Left MCA } \\
\text { (TAMM) }\end{array}$ & $25 \begin{array}{c}120 \\
(102-130)\end{array}$ & -0.266 & 0.199 & -0.15 & 0.473 \\
\hline $\begin{array}{l}\text { Zonare Left MCA } \\
\text { (TAMM) }\end{array}$ & $25 \begin{array}{c}115 \\
(100-125)\end{array}$ & -0.494 & 0.012 & 0.292 & 0.157 \\
\hline
\end{tabular}

The velocities from the right and left MCA were analyzed separately, and interhemispheric differences were assessed. A statistically significant correlation between the right and left MCA was demonstrated using both pieces of equipment. Additionally, ICC statistics demonstrated a good level of agreement between the two pieces of equipment; see Table 3 for details.

\section{Table 3.}

\section{Correlation of readings from two instruments}

Spearman's Correlation Coefficient between right and left TAMM velocity

\begin{tabular}{lcc}
\hline & rho & $P$-value \\
Philips IU-22 & 0.675 & $<0.001$ \\
Zonare Z-One & 0.687 & $<0.001$ \\
\hline
\end{tabular}

ICC between both instruments measuring TAMM velocity in the right and left MCA

\begin{tabular}{lccc}
\hline & ICC & $95 \%$ CI & $P$-value \\
Right MCA & 0.366 & $(-0.066-0.683)$ & 0.005 \\
Left MCA & 0.558 & $(0.213-0.778)$ & 0.001 \\
\hline
\end{tabular}

The PSV measurements from the two ultrasound machines and the flow-simulating phantom are presented in Table 4, which shows the percentage error of these measurements. 
Table 4.

Descriptive statistics of PSV $(\mathrm{cm} / \mathrm{sec})$ recorded using the Philips $I U-22$ ultrasound machine with a linear and phased array probe and the Zonare Z-One using a phased array probe

\begin{tabular}{lcc}
\hline & $\begin{array}{c}\text { Philips IU-22 Phased } \\
\text { Array }\end{array}$ & $\begin{array}{c}\text { Zonare Z-One Phased } \\
\text { Array }\end{array}$ \\
\hline $\mathrm{n}$ & 25 & 25 \\
\hline Mean $\pm \mathrm{SD}$ & $83.44 \pm 5.55$ & $56.64 \pm 2.08$ \\
\hline $95 \% \mathrm{CI}$ & $81.15-85.73$ & $58.78-60.50$ \\
\hline Median $(\mathrm{IQR})$ & $84(78-89)$ & $59(59-61)$ \\
\hline$\%$ Error & 31 & 53 \\
\hline
\end{tabular}

Bland-Altman analysis (Figures 2 and 3) shows that the velocities recorded in the right and left MCA are within the limits of agreement, suggesting agreement between Philips IU-22 and Zonare Z-One. However, the 95\% confidence intervals are wide, reflecting the small sample size and the significant variation of the differences. There is a divergence as the velocities increase.

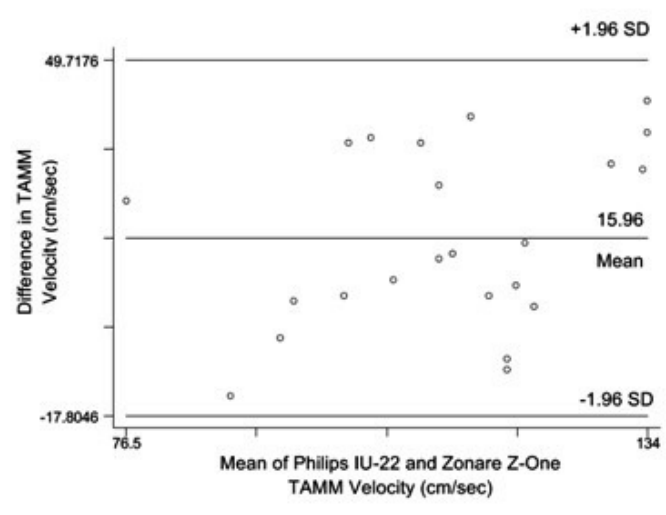

Fig. 2. Right MCA: Bland-Altman analysis: Agreement between TAMM velocity $(\mathrm{cm} / \mathrm{sec})$ measured by Philips $I U-22$ and Zonare Z-One showing the 95\% limits of agreement -17.8 to 49.8 (1.96 Standard Deviation)

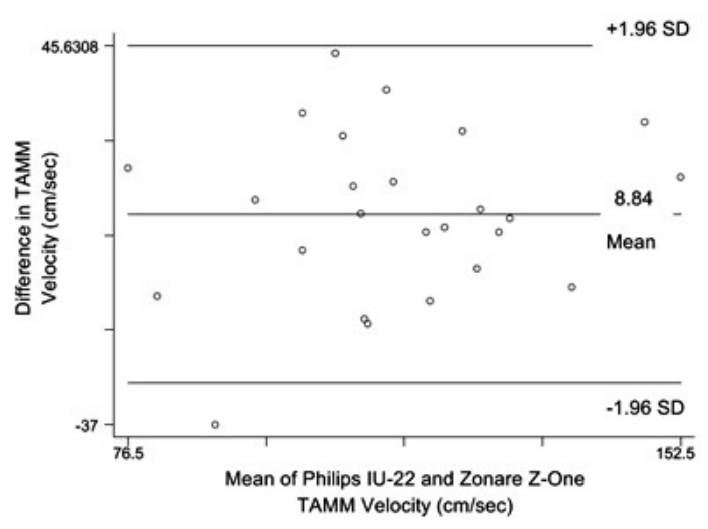

Fig. 3. Left MCA: Bland-Altman analysis: Agreement between TAMM velocity $(\mathrm{cm} / \mathrm{sec})$ measured by Philips $I U-22$ and Zonare Z-One showing the 95\% limits of agreement -17.8 to 49.8 (1.96 Standard Deviation)

Stroke risk categorization according to TAMM velocities in both MCAs measured in both instruments were in full agreement, and all readings were normal in the 25 study participants.
TCCD with sample volume in the MCA using the Zonare Z-One machine and the Philips IU-22 machine are presented in Images 1 and 2 .

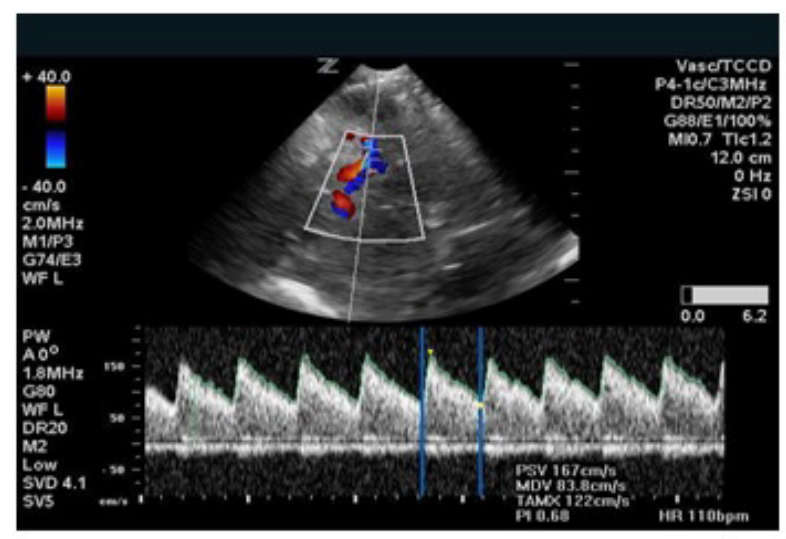

Image 1. TCCD with sample volume in the MCA using the Zonare Z-One machine. Spectral waveform is enclosed in an envelope with measurement over one cardiac cycle giving PSV, Minimum Diastolic Velocity (MDV) and Time-Averaged Max (TAMX) velocity.

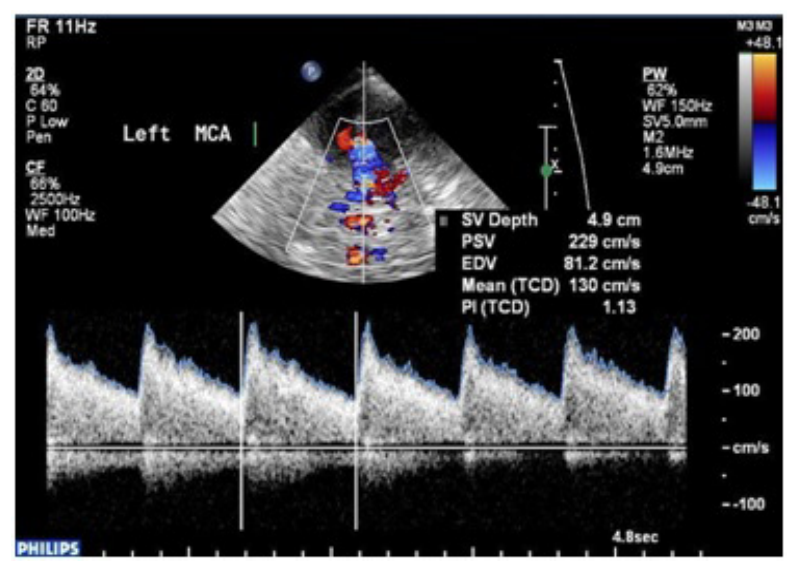

Image 2. TCCD with sample volume in the MCA using the Philips IU-22 machine. Spectral waveform is enclosed in an envelope with measurement over one cardiac cycle giving PSV, End Diastolic Velocity (EDV) and Mean (TCD) which is the TAMM velocity. The sample volume (SV) depth was also recorded.

\section{Discussion}

Moderate values of correlation between TAMM velocities in the right and left MCA of children with SCD were found, indicating little interhemispheric difference. This finding is supported by a previous study. ${ }^{(13)}$ There was a moderate negative correlation between age and TAMM velocity, reflecting the known inverse relationship between blood flow TCD velocities and age. ${ }^{(4)}$ However, unlike previous studies, ${ }^{(14)}$ no significant correlation was found between TAMM and hemoglobin levels. This may be explained by the fact that recently transfused children were not excluded from the study, and recent transfusions can reduce flow velocity. ${ }^{(15)}$

This study has demonstrated a moderate correlation in the TAMM velocity readings in the right and left MCA between the two ultrasound machines in children with SCD. 
This indicates an association between the TAMM velocities but does not necessarily imply agreement. ${ }^{(16)}$

Several factors may explain minor differences in readings between the two instruments. Firstly, it is recognized that TCCD ultrasonography does have limitations because it is operator-dependent. The difference between the two machines may be accounted for by minimal differences in probe position and sample volume placement, resulting in significant differences in velocity measurements. ${ }^{(11)}$ This may account for some discrepancy in the readings; however, the effort was made to minimize this by measuring the velocity at the same sample volume depth in the vessel. Variability in velocity measurements can be introduced because velocity measurements depend on the site where a particular vessel segment is sampled, and the blood flow distribution may not be uniform across curved and tortuous arteries. ${ }^{(17)}$ Discrepancies can be due to different sampling sites as the distribution of blood flow across the artery may not be uniform and there are variations in anatomy and tortuosity of the MCA. ${ }^{(11)}$

No angle correction was used during this study as it is assumed that the angle of insonation between the ultrasound beam and the direction of the blood flow in the MCA is close to $0^{\circ}$ so that the velocity can be measured accurately. ${ }^{(18)}$ However, differences in anatomy of the vessels may introduce variability and errors. TCCD allows for visualization of the vessels and placement of the sample volume to try to keep the site of sampling the same. However, human error could have been a factor due to the differences in the machines. On the Zonare Z-One portable machine, the operator rated the ease of obtaining the TAMM velocity measurement in the MCA in terms of the adequacy of view of the circle of Willis and visualization of the distal MCA. The study carried out by Lui et al. found that the waveform display size was important and could be a factor when using the Zonare Z-One as the display size and resolution are not comparable to the Philips IU-22. The transducers are of similar size so that the positioning in the temporal window was consistent. ${ }^{(19)}$

The MCA was chosen for the comparison because previous studies have suggested that there is less variation in velocities obtained in this vessel due to its anatomy and position relative to the ultrasound beam. ${ }^{(12,20)}$ The measurement of the Doppler shift is dependent on the flow velocity and the angle of insonation. In this situation, the insonation angle is unknown but is assumed to be close to zero due to the anatomy of the MCA. Angle-corrected TCCD velocities are not widely used because it has been suggested that they can overestimate the risk of stroke. ${ }^{(21)}$

The examinations were performed by the same operator, which removed inter-operator variability. The gain was adjusted to ensure optimal signal-to-noise ratio with the precise automatic tracing of the waveforms, and no manual measurements of velocity were made during the study. We thought that this was less time-consuming as well as more precise and consistent. This adjustment did not vary between machines in an effort to reduce discrepancies in velocity measurements. However, it is not known if there is any discrepancy between the automatic tracing features of the ultrasound machines, so this may need further investigation.
Velocity measurements may also be influenced by inherent differences in signal processing.

Although the machines were shown to significantly correlate, Bland-Altman analysis demonstrated wide lines of agreement and variation in differences. It also suggested that as TAMM velocities increase, the variation may also increase. Only one value was greater than two standard deviations, indicating that the repeatability is acceptable.

However, the direct comparisons must be treated with caution. This study focused on the agreement between the two ultrasound machines, rather than the accuracy of the portable ultrasound machine itself. Philips IU-22 was the assumed standard; however, the phantom showed that this standard may also underestimate velocities (data not shown). Further investigation of these differences may require a string phantom, which produces a well-defined velocity ${ }^{(21)}$ and is recommended for annual quality control by the Institute of Physics and Engineering in Medicine. ${ }^{(22)}$

Physiological factors in the children, such as $\mathrm{CO}_{2}$ and hemoglobin, were minimized by performing the measurements on the same day to reduce hemodynamic and hematological differences. However, it should be taken into account that the study was performed such that the examination on the Zonare Z-One was carried out after the examination using Philips IU22. Some of the children were becoming restless during this second examination and may have reduced the opportunity to optimize the examination.

A further limitation is the relatively small sample size in the study, which was related to the difficulties of recruiting children with SCD. However, we believe that our sample was sufficient to provide a valid comparison of the machines. The examinations were performed by the same operator, which may be seen as a limitation because the individual may have been more proficient with a particular machine and was not blinded to the velocities recorded during the previous examination.

\section{Conclusion}

The purpose of this pilot study was to evaluate the use of a portable ultrasound machine by comparison with an established, laboratory-based ultrasound machine. Both machines were found to underestimate PSV using a flow phantom. This study demonstrated complete agreement in stroke risk categorization between the two instruments. As a pilot study, it confirms the feasibility and clinical significance of this investigation. The children identified to be at a higher risk by Philips IU-22 were not identified by Zonare Z-One. A larger sample size with a greater range in TAMM velocities is needed to investigate further the correlation between different ultrasound machines at extremes of measurement, such as very high or very low velocities. Further evidence is needed to establish the implications these differences may have on screening.

\section{Acknowledgments}

This project was supported by the Deanship of Scientific Research at Prince Sattam bin Abdulaziz University. 


\section{Competing Interests} interests.

The authors declare that they have no competing

\section{References}

1. Piel FB, Steinberg MH, Rees DC. Sickle Cell Disease. N Engl J Med. 2017 Apr 20;376(16):1561-1573. doi: 10.1056/ NEJMra1510865.

2. Ohene-Frempong K, Weiner SJ, Sleeper LA, Miller ST, Embury S, Moohr JW, Wethers DL, Pegelow CH, Gill FM. Cerebrovascular accidents in sickle cell disease: rates and risk factors. Blood. 1998 Jan 1;91(1):288-94.

3. Adams RJ, McKie VC, Carl EM, Nichols FT, Perry R, Brock K, McKie K, Figueroa R, Litaker M, Weiner S, Brambilla D. Long-term stroke risk in children with sickle cell disease screened with transcranial Doppler. Ann Neurol. 1997 Nov;42(5):699-704. doi: 10.1002/ana.410420505.

4. Adams RJ, McKie VC, Hsu L, Files B, Vichinsky E, Pegelow C, Abboud M, Gallagher D, Kutlar A, Nichols FT, Bonds DR, Brambilla D. Prevention of a first stroke by transfusions in children with sickle cell anemia and abnormal results on transcranial Doppler ultrasonography. N Engl J Med. 1998 Jul 2;339(1):5-11. doi: 10.1056/NEJM199807023390102. 5. Bernaudin F, Verlhac S, Arnaud C, Kamdem A, Chevret S, Hau I, Coïc L, Leveillé E, Lemarchand E, Lesprit E, Abadie I, Medejel N, Madhi F, Lemerle S, Biscardi S, Bardakdjian J, Galactéros F, Torres M, Kuentz M, Ferry C, Socié G, Reinert P, Delacourt C. Impact of early transcranial Doppler screening and intensive therapy on cerebral vasculopathy outcome in a newborn sickle cell anemia cohort. Blood. 2011 Jan 27;117(4):1130-40; quiz 1436. doi: 10.1182/ blood-2010-06-293514.

6. Cherry MG, Greenhalgh J, Osipenko L, Venkatachalam M, Boland A, Dundar Y, Marsh K, Dickson R, Rees DC. The clinical effectiveness and cost-effectiveness of primary stroke prevention in children with sickle cell disease: a systematic review and economic evaluation. Health Technol Assess. 2012;16(43):1-129. doi: 10.3310/hta16430.

7. UK Forum on Haemoglobin Disorders. Transcranial Doppler Scanning for Children with Sickle Cell Disease Standards and Guidance UK2016 [06/03/2017]. Available from: http://www.haemoglobin.org.uk/wp-content/uploads/2016/10/ tcdstandards.pdf.

8. West Midlands Quality Review Service. Quality Standards Health Services for people with Haemoglobin Disorders UK2014. Available from: http://www.wmqrs.nhs. uk/publications.

9. Jones AM, Seibert JJ, Nichols FT, Kinder DL, Cox K, Luden J, Carl EM, Brambilla D, Saccente S, Adams RJ. Comparison of transcranial color Doppler imaging (TCDI) and transcranial Doppler (TCD) in children with sickle-cell anemia. Pediatr Radiol. 2001 Jul;31(7):461-9. doi: 10.1007/ s002470100427.

*Corresponding author: Dr Mohammed J. Alsaadi MSc, PhD Radiology and Medical Imaging Department, College of Applied Medical Sciences, Prince Sattam Bin Abdulaziz University, Alkharj, Saudi Arabia.E-mail: m.alsaadi@psau.edu.sa
10. Telfer P, Dwan K, Simmons A, Evanson J, Gadong N, Newell K, Tangayi S, Leigh A, Tsouana E, Hemmaway C, Kaya B. Transcranial Doppler Screening in a Regional Care Network for Sickle Cell Disease in the United Kingdom. J Pediatr Hematol Oncol. 2016 Oct;38(7):517-24. doi: 10.1097/ MPH.0000000000000633.

11. McCarville MB, Li C, Xiong X, Wang W. Comparison of transcranial Doppler sonography with and without imaging in the evaluation of children with sickle cell anemia. AJR Am J Roentgenol. 2004 Oct;183(4):1117-22. doi: 10.2214/ ajr.183.4.1831117.

12. Dunn G, Everitt B. Clinical Biosatistics: an Introduction to Evidence Based Medicine. London: Edward Arnold; 1995. 13. Arkuszewski M, Krejza J, Chen R, Kwiatkowski JL, Ichord R, Zimmerman R, Ohene-Frempong K, Desiderio L, Melhem ER. Sickle cell disease: reference values and interhemispheric differences of nonimaging transcranial Doppler blood flow parameters. AJNR Am J Neuroradiol. 2011 Sep;32(8):144450. doi: 10.3174/ajnr.A2529.

14. Pavlakis SG, Rees RC, Huang X, Brown RC, Casella JF, Iyer RV, Kalpatthi R, Luden J, Miller ST, Rogers ZR, Thornburg CD, Wang WC, Adams RJ; BABY HUG Investigators. Transcranial doppler ultrasonography (TCD) in infants with sickle cell anemia: baseline data from the BABY HUG trial. Pediatr Blood Cancer. 2010 Feb;54(2):256-9. doi: $10.1002 /$ pbc. 22282.

15. Kwiatkowski JL, Yim E, Miller S, Adams RJ; STOP 2 Study Investigators. Effect of transfusion therapy on transcranial Doppler ultrasonography velocities in children with sickle cell disease. Pediatr Blood Cancer. 2011 May;56(5):777-82. doi: 10.1002/pbc.22951.

16. Bland JM, Altman DG. Applying the right statistics: analyses of measurement studies. Ultrasound Obstet Gynecol. 2003 Jul;22(1):85-93. doi: 10.1002/uog.122.

17. Bulas DI, Jones A, Seibert JJ, Driscoll C, O’Donnell R, Adams RJ. Transcranial Doppler (TCD) screening for stroke prevention in sickle cell anemia: pitfalls in technique variation. Pediatr Radiol. 2000 Nov;30(11):733-8. doi: $10.1007 / \mathrm{s} 002470000317$.

18. Krejza J, Rudzinski W, Pawlak MA, Tomaszewski M, Ichord R, Kwiatkowski J, Gor D, Melhem ER. Anglecorrected imaging transcranial doppler sonography versus imaging and nonimaging transcranial doppler sonography in children with sickle cell disease. AJNR Am J Neuroradiol. 2007 Sep;28(8):1613-8. doi: 10.3174/ajnr.A0591.

19. Lui EY, Steinman AH, Cobbold RS, Johnston KW. Human factors as a source of error in peak Doppler velocity measurement. J Vasc Surg. 2005 Nov;42(5):972-9. doi: 10.1016/j.jvs.2005.07.014.

20. WalkerA, Olsson E, Wranne B, Ringqvist I,Ask P. Accuracy of spectral Doppler flow and tissue velocity measurements in ultrasound systems. Ultrasound Med Biol. 2004 Jan;30(1):12732. doi: 10.1016/j.ultrasmedbio.2003.08.020.

21. Browne JE. A review of Doppler ultrasound quality assurance protocols and test devices. Phys Med. 2014 Nov;30(7):742-51. doi: 10.1016/j.ejmp.2014.08.003.

22. Krejza J, Swiat M, Pawlak MA, Oszkinis G, Weigele J, Hurst RW, Kasner S. Suitability of temporal bone acoustic window: conventional TCD versus transcranial color-coded duplex sonography. J Neuroimaging. 2007 Oct;17(4):311-4. doi: 10.1111/j.1552-6569.2007.00117.x. 